\title{
LINE BLANKETING IN REAL STELLAR ATMOSPHERES
}

\author{
P. L. BERNACCA \\ Asiago Observatory, Italy
}

\begin{abstract}
Ultraviolet observations of line blanketing in the spectrum variables $\alpha \mathrm{Scl}$ and $\alpha^{2} \mathrm{CVn}$ show that the usual procedure of allowing line blocking in model atmospheres has to be revised. It is also suggested that the region around $2900 \AA$ could be safely used for determining effective temperatures.
\end{abstract}

Everybody knows that a blanketed model atmosphere is one where the line opacities are included in the computation of the emergent continuum flux. Blanketed model atmospheres exist that include the hydrogen lines (Klinglesmith, 1971) as well as the strongest ultraviolet lines (e.g. Bradley and Morton 1969, and references therein). The procedure followed in these calculations makes use of the radiative flux conservation relation

$$
\int_{0}^{\infty} F(\lambda, \tau) \mathrm{d} \lambda=\text { const } .
$$

The constancy of the integrated flux is maintained by adjusting the temperature structure of the atmospheres, that is the law $T(\tau)$, so that a blanketed model corresponds to an unblanketed one of higher effective temperature. In other words blanketing yields a higher continuum between the lines.

I think it is of interest to consider some recent observations made by OAO-II in the ultraviolet, which give us a hint as to how blocking and backwarming works in a real atmosphere. The cases of interest here are those of the stars $\alpha \mathrm{Scl}$ and $\alpha^{2} \mathrm{CVn}$.

The star $\alpha \mathrm{Scl}$ is a helium-weak star of Population I and it was observed in the ultraviolet region by Bernacca and Molnar (1972) (BM). Most recently Norris (1971) has obtained scanner observations of its continuum down to $\lambda 3400$. More detailed information on the star can be found in the above two papers. In Figure 1 the photometry data from BM and the scanner observations from Norris are presented together with the run of the flux $F_{\lambda}$ according model calculations by Klinglesmith (1971). The star $\alpha \mathrm{Scl}$ has been given a $T_{\text {eff }}=14400$ by using ultraviolet blanketed models (Norris, 1971) and one sees indeed from the figure that the ground based observations (crosses) fit reasonably well to a hydrogen line blanketed model of $T_{\text {eff }}=14000$.

If we consider the ultraviolet observations made on January 23, 1971 (open circles) we can say that the effective temperature of $\alpha \mathrm{Scl}$ is more close to $16000 \mathrm{~K}$. On July 10, 1970 (filled circles), the energy output from the star at $2380 \AA$ and $2460 \AA$ is decreased and at the same time an increase of luminosity appears at $4250 \AA$. This behaviour has been explained by BM as a result of variable line blocking in the $2400 \AA$ region which redistributes energy (backwarming) longward of the Balmer discontinuity. The blocking can be attributed to the strengthening of $\mathrm{Ti}$ II and $\mathrm{Sr}$ II lines which are known to be unusual in this star. 


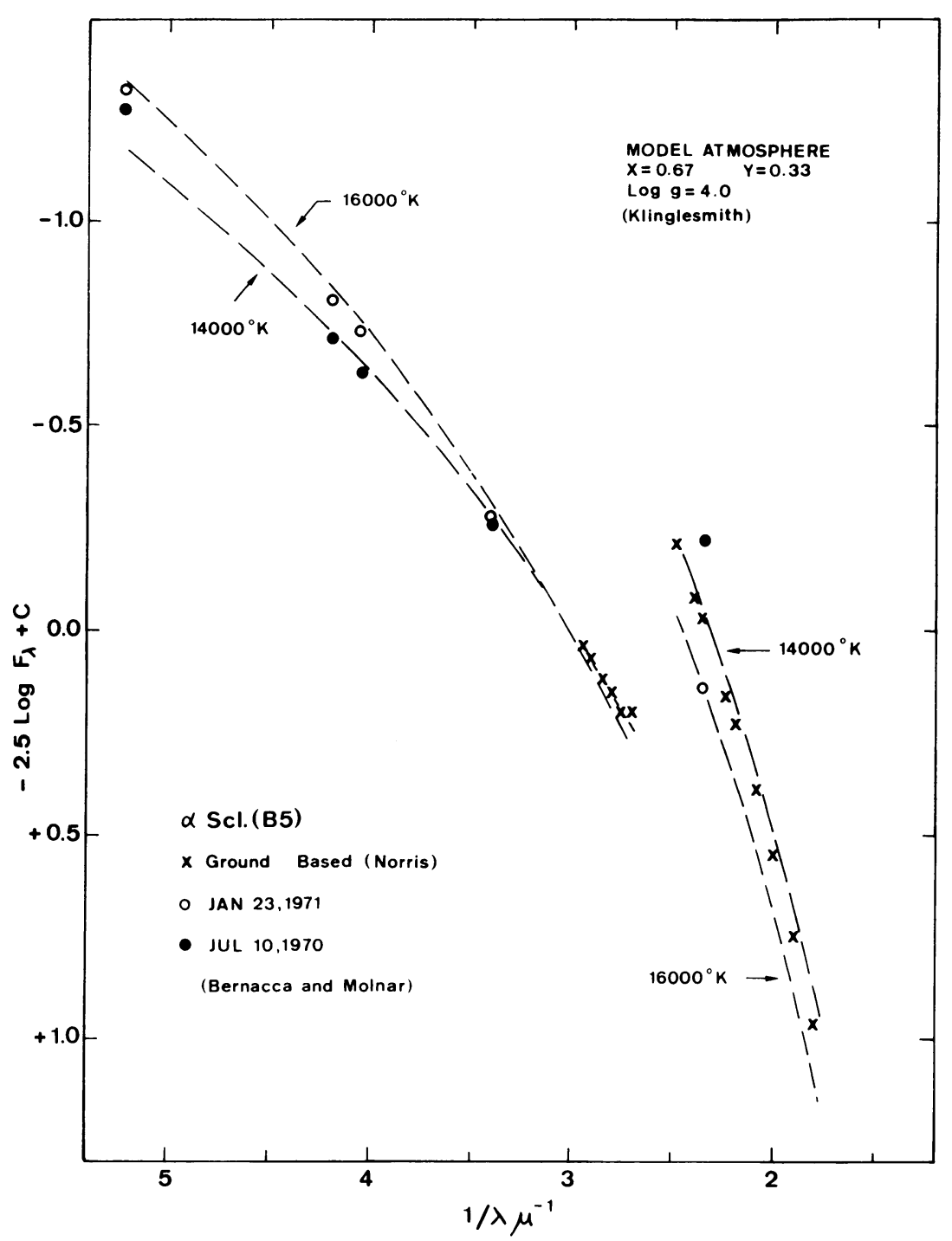

Fig. 1. Ultraviolet and ground based observations of $\alpha \mathrm{Scl}$ are compared with hydrogen line blanketed models. The flux is normalized at $1 / \lambda=3.0 \mu^{-1}$. The ground based observations by Norris (1971) from $1 / \lambda=1.8 \mu^{-1}$ to $1 / \lambda=2.95 \mu^{-1}$ have been easily extrapolated at $3.0 \mu^{-1}$.

From the figure we may infer that it is possible that Norris' observations were made at a phase where the atmosphere of the star was similar to that observed on July 10 , 1970.

The second remarkable object is the Ap variable $\alpha^{2} \mathrm{CVn}$ studied in the ultraviolet by Molnar (1972), to whom we refer for the relevant information concerning this star. It is sufficient here to point out that the star $\alpha^{2} \mathrm{CVn}$ is a light variable in $U, B, V$, and 
at $3317 \AA, 2460 \AA, 2380 \AA, 1910 \AA, 1550 \AA, 1430 \AA$, and $1330 \AA$. The star has a constant luminosity at $2945 \AA$ and $2985 \AA$. The intensity of the metal lines varies periodically with a period of 5.47 days.

All the light curves in the region $\lambda \lambda 1910-2460$ have a minimum at phase 0.0 when the rare earths have maximum lin strength in the spectrum of the star. At the same phase the $V$ light curve has a maximum. All the light curves below $1910 \AA$ have a minimum at phase $0.1-0.2$ when the $U, B$ and $\lambda 3317$ light curves show a maximum.

This is interpreted as variable blanketing: blocking of radiation between 2000 and $2600 \AA$ and consequent backwarming in the $V$ band; blocking shortward of $2000 \AA$ and backwarming in the $U$ and $B$ bands and in the near Balmer continuum. The behaviour of $\alpha^{2} \mathrm{CVn}$ is similar to that of $\alpha \mathrm{Scl}$ as far as the line blocking between $2000 \AA$ and $2600 \AA$ is concerned.

Now, how does line blanketing behave in stars with normal spectra? Underhill (1971) has shown the existence of a large amount of line blocking in the ultraviolet spectrum of main-sequence B-type stars and we should worry about any fitting of the observations to classical blanketed models. There is no reason to exclude that $\alpha^{2} \mathrm{CVn}$ and $\alpha$ Scl indicate how line blanketing works actually in any stellar atmosphere. Instead of increasing the continuum between the lines, it is conceivable that the radiation substracted in a certain region of the spectrum is degraded longward. Where it is reemitted can be well indicated by the two peculiar stars presented above.

A minor conclusion is that the region around $2900 \AA$ seems to be free from both blocking and backwarming effects so that it could be safely used to give a star a $T_{\text {eff }}$.

\section{References}

Bernacca, P. L. and Molnar, M. R.: 1972, Astrophys. J. 178, No. 1.

Bradley, P. T. and Morton, D. C.: 1969, Astrophys. J. 156, 687.

Klinglesmith, D. A.: 1971, NASA SP-3065.

Norris, J.: 1971, Astrophys. J. Suppl. 23, 213.

Underhill, A. B.: 1971, Proceedings OAO-Symposium, Amherst, Mass., NASA-SP-310, 367.

\section{DISCUSSION}

Jaschek: Your data provide the first reliable basis for calling $\alpha$ Scl a spectrum variable, confirming thus earlier suspicions.

Schild: In noting a $2000 \mathrm{~K} T_{\text {eff }}$ difference between a model atmosphere and OAO II flux of $\alpha$ Scl, you mention the models of Klinglesmith; can you tell how line blocking was treated in the Klinglesmith model?

Bernacca: Klinglesmith's models are hydrogen blanketed models and the line blocking is treated in the usual way, that is by imposing the constancy of the integrated flux at each optical depth. 